International Journal of Pure and Applied Mathematics

Volume 89 No. 3 2013, 295-303

ISSN: 1311-8080 (printed version); ISSN: 1314-3395 (on-line version)

url: http://www.ijpam.eu

doi: http://dx.doi.org/10.12732/ijpam.v89i3.1

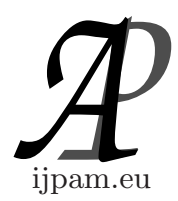

\title{
ON THE INVARIANT SUPSPACE PROBLEM
}

\author{
C. Tonyalı ${ }^{1}$, E. Bayram ${ }^{2} \S$ \\ ${ }^{1}$ Department of Mathematics \\ Gazi University, Ankara, TURKEY \\ ${ }^{2}$ Department of Mathematics \\ Namik Kemal University \\ Tekirdag, TURKEY
}

\begin{abstract}
In this study, we present invariant subspaces (subideals) for a class of operators (positive operators) related to M-weakly and L-weakly compact operators. Principally, these invariant subspaces can be conceivable for all operators that commutes with any M-weakly or L-weakly compact operator.
\end{abstract}

AMS Subject Classification: 47A15, 47B65, 47B60, 46B42

Key Words: invariant subspace, L-weakly compact operator, M-weakly compact operator

\section{Introduction}

The invariant subspace problem can be described by "when does a bounded operator on a Banach space of dimensional at least two have a non-trivial closed invariant subspace?". Suppose that X is a Banach space and T is a bounded operator defined on X. A subspace $\mathrm{W}$ of $\mathrm{X}$ is called non-trivial invariant subspace under $\mathrm{T}$ if $W \neq\{0\}, W \neq X$ and $T(W) \subseteq W$. There is a great deal of literature about invariant subspace problem. The problem was solved for compact operators, or for operators related to compact operators, and other some classes of operators on Banach spaces or on Banach lattices, see [1],[3], [4].

Received: September 27, 2012

(C) 2013 Academic Publications, Ltd.

$\S_{\text {Correspondence author }}$ url: www.acadpubl.eu 
The aim of our investigations is whether or not all $L$-weakly and $M$-weakly compact operators and some bounded operator classes related to these operator classes defined on Banach lattices have a non-trivial closed invariant subspace (or subideal). These two classes of bounded operators are subclasses of weakly compact operators but they need not be compact operator and vice versa. $L$-weakly and $M$-weakly compact operators were introduced by P. MeyerNieberg in [5].

In this study, we shall prove that every $M$-weakly compact operator defined on Banach lattice $E$ such that the prepolar ${ }^{\circ} E_{a}^{\prime}$ of the order continuous part of the norm dual space $E^{\prime}$ does not equal to $\{0\}$ have a non-trivial closed common invariant subideal. Depending on this result, under same conditions we shall show all bounded operators that commutes with any $M$-weakly compact operator have a non-trivial closed invariant subspace. Following this, we shall give similar results for all bounded operators that commutes with any $L$-weakly or $M$-weakly compact operator defined on Banach lattice $E$ if $E$ or its norm dual space $E^{\prime}$ have a sublattice such that lattice isomorphic to $L_{1}(\mu), \mu$ is a measure without atom. Furthermore, as an analogous to these results, we shall see that all positive operators that commutes with a positive $M$-weakly or $L$-weakly compact operator on a Banach lattice $E$ under same conditions have a non-trivial closed invariant subideal.

\section{Preliminaries}

Before we present our results, let us mention about definitions and notations, used in this paper. Throughout this paper, unless otherwise state, $E$ will denote an infinite dimensional separable real Banach lattice with norm dual space $E^{\prime}$. In the rest of this article, by the term "an operator" between two Banach lattices, we shall mean " a linear norm bounded operator" and all operators that we are looking for its invariant subspaces will be assumed to be nonscalar, non-zero, one-to-one and have dense range. An operator $T$ from the Banach lattice $E$ into the Banach lattice $F$ is called positive if $T\left(E^{+}\right) \subseteq F^{+}$. $T S$ means composition of the operators $T$ and $S$. So, for $k \in \mathbb{N}^{+} S^{k}$ means composition of the operator $S$ with itself k times. We refer to [2], [6] and [8] for any unexplained terms from Banach lattice theory and for further details on the theory of invariant subspaces see [1], [7].

Recall that a non-empty bounded subset $A$ of the Banach lattice $E$ is said to be $L$-weakly compact if $\left\|x_{n}\right\| \rightarrow 0$ for every disjoint sequence $\left(x_{n}\right)$ in the solid hull of $A$. A bounded linear operator $T$ from a Banach space $X$ into $E$ is called 
$L$-weakly compact if $T\left(B_{X}\right)$ is $L$-weakly compact in $E$, where $B_{X}$ denotes the closed unit ball of $X$. A bounded linear operator from $E$ into $X$ is $M$-weakly compact if $\left\|T x_{n}\right\| \rightarrow 0$ as $n \rightarrow \infty$ for every disjoint sequence $\left(x_{n}\right)$ in $B_{E}$. In [5], P. Meyer-Nieberg showed that an operator defined between two Banach lattice is $L$-weakly ( $M$-weakly) compact if and only if its adjoint operator is $M$-weakly ( $L$-weakly) compact and also that $L$-weakly and $M$-weakly compact operators are weakly compact operators. It is known that

$$
E^{a}=\{x \in E: \text { every monotone sequence in }[0,|x|] \text { is convergent }\}
$$

is the maximal closed order ideal in $E$ on which the induced norm is order continuous and that any $L$-weakly compact subset is contained in $E^{a}$ ([6], page 92 and page 212). Recall that a Banach lattice $E$ is said to have an order continuous norm if $x_{\alpha} \downarrow 0$ in $E$ implies $\left\|x_{\alpha}\right\| \downarrow 0$. For example the Banach lattices $L^{1}[0,1], \ell_{1}, c_{0}$ have order continuous norm but the Banach lattices $L^{\infty}[0,1], \ell_{\infty}, c$ does not have order continuous norm.

Let $E$ be a Riesz space. A linear functional $f: E \rightarrow \mathbb{R}$ is said to be positive whenever $f(x) \geq 0$ holds for each $x \in E^{+}$. Also, a linear functional $f$ is called order bounded if $f$ maps order bounded subsets of $E$ onto bounded subsets of $\mathbb{R}$. The vector space $E^{\sim}$ of all order bounded linear functionals on $E$ is called the order dual of $E$. A positive functional $f$ on $E$ is order continuous if and only if $x_{\alpha} \downarrow 0$ in $E$ implies $f\left(x_{\alpha}\right) \downarrow 0$. All order continuous linear functionals on $E$ are denoted by $E_{n}^{\sim}$. Clearly, we have $E_{n}^{\sim} \subseteq E^{\sim}$ ([2], Page 56).

Let $E$ be a Banach lattice. If $A$ is a subset of $E$, then its polar $A^{\circ}$ is defined by $A^{\circ}=\left\{x^{\prime} \in E^{\prime}:\left|x^{\prime}(x)\right| \leq 1\right.$ for every $\left.x \in A\right\}$. $A^{\circ}$ is a convex, circled and $\sigma\left(E^{\prime}, E\right)$-closed subset. If $B$ is a subset of the dual space $E^{\prime}$ then we call prepolar ${ }^{\circ} B$ defined by ${ }^{\circ} B=\left\{x \in E:\left|x^{\prime}(x)\right| \leq 1\right.$ for every $\left.x^{\prime} \in B\right\}$. If $B \subseteq E^{\prime}$ is an ideal, then prepolar ${ }^{\circ} B$ is an ideal and we see that ${ }^{\circ} B=$ $\left\{x \in E: x^{\prime}(x)=0\right.$ for every $\left.x^{\prime} \in B\right\}$. Then, according to definitions we have $A \subseteq{ }^{\circ}\left(A^{\circ}\right)$ and $B \subseteq\left({ }^{\circ} B\right)^{\circ}([2]$, page 140$)$.

\section{Main Results}

We know that the maximal ideal $E^{a}$ is non-trivial closed common invariant subideal for all non-zero L-weakly compact operators defined on Banach lattice $E$ without order continuous norm. As a dual version of this result, we can consider the following result.

Proposition 1. Let $E$ be a Banach lattice such that the prepolar ${ }^{\circ} E_{a}^{\prime}$ of the order continuous part of the norm dual space $E^{\prime}$ does not equal to $\{0\}$. 
Then, for every non-zero $M$-weakly compact operator defined on $E$ there exists a non-trivial closed common invariant subideal.

Proof. Assume that ${ }^{\circ} E_{a}^{\prime} \neq\{0\}$ holds and the operator $T: E \rightarrow E$ is any non-zero $M$-weakly compact operator. Then, the adjoint operator $T^{\prime}: E^{\prime} \rightarrow$ $E^{\prime}$ is a $L$-weakly compact operator. Hence, we get $T^{\prime}\left(B_{E^{\prime}}\right) \subseteq E_{a}^{\prime}$, where $B_{E^{\prime}}$ denotes the closed unit ball of dual space $E^{\prime}$ since any $L$-weakly compact set of $E^{\prime}$ is contained in $E_{a}^{\prime}$. Since we assume ${ }^{\circ} E_{a}^{\prime} \neq\{0\}$ let us take any $x \in{ }^{\circ} E_{a}^{\prime}$ such that $x \neq 0$. For all non-zero element $f$ in $B_{E^{\prime}}, f(T x)=T^{\prime} f(x)=0$ since $T^{\prime} f \in E_{a}^{\prime}$. It means that for all elements $x$ in ${ }^{\circ} E_{a}^{\prime}, T x=0$ holds. Therefore, ${ }^{\circ} E_{a}^{\prime}$ is $T$-invariant. Furthermore, ${ }^{\circ} E_{a}^{\prime}$ is norm closed subideal since ${ }^{\circ} E_{a}^{\prime}$ is $\sigma\left(E, E^{\prime}\right)$-closed, convex and $E_{a}^{\prime}$ is a ideal in $E^{\prime}([2]$, Page 140 and 171). On the other hand, suppose that ${ }^{\circ} E_{a}^{\prime}=E$. Then, $E_{a}^{\prime} \subseteq\left({ }^{\circ} E_{a}^{\prime}\right)^{\circ}=E^{\circ}=\{0\}$ implies $E_{a}^{\prime}=\{0\}$. Hence, from the inclusion $T^{\prime}\left(B_{E^{\prime}}\right) \subseteq E_{a}^{\prime}$, we obtain that $T^{\prime}=0$ and so $T=0$. This is a contradiction. Then, ${ }^{\circ} E_{a}^{\prime} \neq E$ must be. We already know that ${ }^{\circ} E_{a}^{\prime} \neq\{0\}$ by the hypothesis. Therefore, the operator $T$ have a non-trivial closed invariant subideal.

There are some situations that the prepolar ${ }^{\circ} E_{a}^{\prime}$ does not equal to $\{0\}$ for the Banach lattice $E$. If the inclusion $E_{a}^{\prime} \subseteq E_{n}^{\sim}$ holds and $E_{a}^{\prime}$ is not order dense in $E_{n}^{\sim}$, then ${ }^{\circ} E_{a}^{\prime} \neq\{0\}$ holds ([10], Corollary 105.12). It is well known if $E$ have order continuous norm then $E^{\prime}=E_{n}^{\sim}$ holds. For instance, the Banach lattice $E=L^{1}[0,1] \oplus c_{0}$ have order continuous norm. On the other hand, $E^{\prime}=L^{\infty}[0,1] \oplus \ell_{1}$ does not have order continuous norm and $E_{a}^{\prime}=\ell_{1}$ is not order dense in $E^{\prime}$. On the contrary, the ideal $\left(\ell_{1}^{\prime}\right)^{a}=c_{0}$ is order dense in $\left(\ell_{1}\right)^{\prime}=\ell_{\infty}([10]$, Page 433).

We can extend the result mentioned above with the help of any non-zero $M$-weakly compact operator defined on a Banach lattice.

Theorem 2. Let $E$ be a Banach lattice such that the prepolar ${ }^{\circ} E_{a}^{\prime}$ does not equal to $\{0\}$ and let the operator $T: E \rightarrow E$ be a non-zero $M$-weakly compact operator. Then, every bounded operator $S: E \rightarrow E$ defined on $E$ such that $T S^{k}$ is a $M$-weakly compact operator for each $k=1,2, \ldots$ have a non-trivial closed invariant subspace.

Proof. Assume that the dual space ${ }^{\circ} E_{a}^{\prime} \neq\{0\}$ holds, the operator $T: E \rightarrow$ $E$ is a non-zero $M$-weakly compact operator and the operator $S: E \rightarrow E$ is a bounded operator such that $T S^{k}$ is $M$-weakly compact operator for each $k=1,2, \ldots$. Let us choose a non-zero element $x_{0}$ in ${ }^{\circ} E_{a}^{\prime}$ since we assume ${ }^{\circ} E_{a}^{\prime} \neq\{0\}$. Again, as we have seen in Proposition 1, it is clear that $T S^{k} x_{0}=0$ holds for each $k=1,2, \ldots$. Since we assume that $S$ is one-to-one we have 
$S^{k} x_{0} \neq 0$ for each $k=1,2, \ldots$. Let $W$ denote the non-zero subspace generated by the set $\left\{x_{0}, S x_{0}, S^{2} x_{0}, \ldots\right\}$. Obviously, $S(\bar{W}) \subseteq \bar{W}$ holds. On the other hand, there exists a non-zero linear functional $f_{0}$ in $E^{\prime}$ such that $T^{\prime} f_{0} \neq 0$ since $T^{\prime} \neq 0$. Hence, $T^{\prime} f_{0}\left(S^{k} x_{0}\right)=f_{0}\left(T S^{k} x_{0}\right)=f_{0}(0)=0$ for each $k=1,2, \ldots$ So, we see that the non-zero linear functional $T^{\prime} f_{0}$ is zero on $\bar{W}$. It means that $\bar{W} \neq E$ holds. Clearly, $\bar{W} \neq\{0\}$. Therefore, $\bar{W}$ is a non-trivial closed $S$-invariant subspace, as desired.

Let $E$ be a Banach lattice, let the operator $T: E \rightarrow E$ is a $M$-weakly compact and let the bounded operator $S: E \rightarrow E$ commutes with $T$. We know that $\lim \left\|T x_{n}\right\|=0$ for every disjoint sequence $\left(x_{n}\right)$ in $B_{E}$ because of the $M$-weakly compactness of $T$. Hence, $S^{k} T$ is a $M$-weakly compact operator for each $k=1,2, \ldots$ since the inequality $\left\|S^{k} T\left(x_{n}\right)\right\| \leq\left\|S^{k}\right\|\left\|T x_{n}\right\|$ holds. On the other hand, $T S^{k}=S^{k} T$ holds for each $k=1,2, \ldots$. Therefore, $T S^{k}$ is a $M$-weakly compact operator for each $k=1,2, \ldots$ Similarly, if the bounded operator $S: E \rightarrow E$ is a operator that preserves disjointness then for each $k=1,2, \ldots T S^{k}: E \rightarrow E$ is a $M$-weakly compact operator where the operator $T$ is $M$-weakly compact. Thus, we obtain next result pursuant to the theorem 1.

Corollary 3. Let $E$ be a Banach lattice such that the prepolar ${ }^{\circ} E_{a}^{\prime}$ does not equal to $\{0\}$. Then, every bounded operator that commutes with any nonzero $M$-weakly compact operator have a non-trivial closed invariant subspace.

For the Banach lattice $E$, if the maximal ideal $E_{a}^{\prime} \neq\{0\}$ it can be defined a $M$-weakly compact operator on $E$. Indeed, if $E_{a}^{\prime} \neq\{0\}$ we can consider the operator $T: E \rightarrow E$ defined by $T(x)=f(x) y$ for each $x \in E$ where the element $f \in\left(E_{a}^{\prime}\right)_{+}$and the element $y$ in $B_{E}^{+}$. Hence, the adjoint operator $T^{\prime}: E^{\prime} \rightarrow E^{\prime}$ is given by $T^{\prime}(g)=g(y) f$ for each $g \in E^{\prime}$. By Theorem 12.12 of [2], $T^{\prime}$ is a $L$-weakly compact operator. Clearly, $T$ is a $M$-weakly compact operator. Similarly, if the maximal ideal $E^{a} \neq\{0\}$ then there exists an $L$-weakly compact operator defined on $E$. Let choose an element $y \in E_{+}^{a}$ and an element $f$ in $B_{E^{\prime}}^{+}$. Define an operator $T: E \rightarrow E$ by $T(x)=f(x) y$ for each $x \in E$ where the element $\mathrm{y}$ is in $E_{+}^{a}$ and the element $f$ is in $B_{E^{\prime}}^{+}$. Since $T\left(B_{E}\right) \subseteq\|f\|[-y, y]$ holds the operator $T$ is $L$-weakly compact by Theorem 12.12 of [2]. If the Banach lattice $E$ does not have order continuous norm then the ideal $E^{a}$ is non-trivial closed common invariant subideal for non-zero $L$-weakly compact operators and we can find non-trivial closed invariant subspaces for bounded operators on $E$ that commutes with any non-zero $L$-weakly compact operator.

As an analogous of Theorem 1, we get the next result, describes positive operators having non-trivial closed invariant subideals. 
Theorem 4. Let $E$ be a Banach lattice such that the prepolar ${ }^{\circ} E_{a}^{\prime}$ does not equal to $\{0\}$, let the operator $T: E \rightarrow E$ be a positive $M$-weakly compact and let the operator $S: E \rightarrow E$ be any positive operator. If for each $k=1,2, \ldots$ $T S^{k}$ is a $M$-weakly compact operator then $S$ have a non-trivial closed invariant subideal.

Proof. Assume that the operator $T: E \rightarrow E$ is a positive $M$-weakly compact and the operator $S: E \rightarrow E$ is positive such that for each $k=1,2, \ldots$ $T S^{k}$ is a $M$-weakly compact operator. Let us choose a non-zero positive element $x_{0}$ in ${ }^{\circ} E_{a}^{\prime}$ since we assume ${ }^{\circ} E_{a}^{\prime} \neq\{0\}$. According to proof of Proposition 1 , we know that for non-zero element $x_{0}$ in ${ }^{\circ} E_{a}^{\prime}$ and for each $k=1,2, \ldots T S^{k} x_{0}=$ 0 holds. Since we assume that the operator $S: E \rightarrow E$ is one-to-one, we have $S^{k} x_{0} \neq 0$ for each $k=1,2, \ldots$. Let $W$ denote the non-zero subideal by the set $\left\{x_{0}, S x_{0}, S^{2} x_{0}, \ldots\right\}$. It means that

$$
W=\left\{y \in E: \exists i \in \mathbb{N} \text { and } \exists \lambda_{1}, \lambda_{2}, \ldots, \lambda_{n} \in \mathbb{R}^{+} \text {with }|y| \leq \sum_{j=1}^{n} \lambda_{j} S^{i+j} x_{0}\right\} .
$$

Hence,

$$
|S y| \leq S|y| \leq S\left(\sum_{j=1}^{n} \lambda_{j} S^{i+j} x_{0}\right)=\sum_{j=1}^{n} \lambda_{j} S^{i+1+j} x_{0} .
$$

It follows that the ideal $W$ is a $S$-invariant ideal. At the same time, it implies $S(\bar{W}) \subseteq \bar{W}$. Since $T^{\prime} \neq 0$, there exists a positive linear functional $f_{0}$ in $E^{\prime}$ such that $T^{\prime} f_{0} \neq 0$. Hence, $T^{\prime} f_{0}\left(S^{k} x_{0}\right)=f_{0}\left(T S^{k} x_{0}\right)=f_{0}(0)=0$ for each $k=1,2, \ldots$. Then, we see that the positive linear functional $T^{\prime} f_{0}$ is zero on $\bar{W}$. It means that $\bar{W} \neq E$ holds. Obviously, $\bar{W} \neq\{0\}$. Therefore, $\bar{W}$ is a non-trivial closed $S$-invariant subideal.

To prove our next result, note that the maximal ideal $E^{a}$ of a Banach lattice $E$ includes all $L$-weakly compact sets. If $\mu$ is a measure without atom then $\left(L^{\infty}(\mu)\right)^{a}=\{0\}([10]$, Page 318). So, the following theorem concerns Banach lattices with a sublattice such that lattice isomorphic to $L^{1}(\mu), \mu$ is a measure without atom.

Theorem 5. Let $E$ be a Banach lattice which have a sublattice such that lattice isomorphic to $L_{1}(\mu), \mu$ is a measure without atom and let the operator $T: E \rightarrow E$ be a non-zero $M$-weakly compact operator. Then every bounded operator $S: E \rightarrow E$ such that $T S^{k}$ is a $M$-weakly compact operator for each $k=1,2, \ldots$ have a non-trivial closed invariant subspace. 
Proof. Let $W$ denote sublattice of $E$ such that lattice isomorphic to $L_{1}(\mu)$, $\mu$ is a measure without atom (we can show like that $W \cong L_{1}(\mu)$ ). If the operator $T: E \rightarrow E$ is a $M$-weakly compact operator then the restricted operator $T_{\left.\right|_{W}}: W \cong L_{1}(\mu) \rightarrow E$ is $M$-weakly compact. So, the adjoint operator $\left(T_{T_{W}}\right): E^{\prime} \rightarrow W^{\prime}$ is $L$-weakly compact. On the other hand, since all $L$-weakly compact sets is contained in $\left(W^{\prime}\right)^{a} \cong\left(L_{\infty}(\mu)\right)^{a}$ we obtain that

$$
\left(T_{\left.\right|_{W}}\right)^{\prime}\left(B_{E^{\prime}}\right) \subseteq\left(W^{\prime}\right)^{a} \cong\left(L^{\infty}(\mu)\right)^{a}=\{0\} .
$$

Thus $\left(T_{\left.\right|_{W}}\right)^{\prime}=0$, so $T$ is zero operator on $W$. Similarly, since the operator $T S^{k}$ : $E \rightarrow E$ is a $M$-weakly compact operators, for each $k=1,2, \ldots T S^{k}\left(x_{0}\right)=0$, for any non-zero element $x_{0}$ in $W$. Let $V$ denote the non-zero subspace generated by the set $\left\{x_{0}, S x_{0}, S^{2} x_{0}, \ldots\right\}$. Clearly, $S(\bar{V}) \subseteq \bar{V}$ holds. Note that $S^{k} x_{0} \neq 0$ holds for each $k=1,2, \ldots$ since we assume that $S$ is one to one operator. On the other hand, there exists a non-zero linear functional $f_{0}$ in $E^{\prime}$ satisfies $T^{\prime} f_{0} \neq 0$ since $T^{\prime} \neq 0$. Therefore, we obtain that $T^{\prime} f_{0}\left(S^{k} x_{0}\right)=f_{0}\left(T S^{k} x_{0}\right)=f_{0}(0)=0$. Hence, the non-zero linear functional $T^{\prime} f_{0}$ is zero on $\bar{V}$. It follows $\bar{V} \neq E$. Clearly, $\bar{V} \neq\{0\}$. Therefore, $\bar{V}$ is non-trivial closed $S$-invariant subspace which we are looking for.

As an example for Banach lattices in Theorem 3, we can give non-discrete AL-spaces.

Theorem 6. Let $E$ be a Banach lattice which have a sublattice such that lattice isomorphic to $L_{1}(\mu), \mu$ is a measure without atom and let the operator $T: E \rightarrow E$ be a positive $M$-weakly compact operator. Then, every positive operator $S: E \rightarrow E$ such that $T S^{k}$ is $M$-weakly compact operator for each $k=1,2, \ldots$ have a non-trivial closed invariant subideal.

Proof. By using technique in the proof of theorem 2 and theorem 3, it is enough to consider that the closed ideal generated by the set $\left\{x_{0}, S x_{0}, S^{2} x_{0}, \ldots\right\}$ for a non-zero positive element $x_{0}$ in sublattice $W$ of $E$ such that lattice isomorphic to $L_{1}(\mu), \mu$ is a measure without atom.

We can state a similar theorem to the previous theorem in consideration of $L$-weakly compact operators.

Theorem 7. Let $E$ be a Banach lattice such that its dual space $E^{\prime}$ have a sublattice such that lattice isomorphic to $L_{1}(\mu), \mu$ is a measure without atom and let the operator $T: E \rightarrow E$ be a non-zero $L$-weakly compact operator. Then, every bounded operator $S: E \rightarrow E$ such that $S^{k} T$ is a $L$-weakly compact operator for each $k=1,2, \ldots$ have a non-trivial closed invariant subspace. 
Proof. Assume that the operator $T: E \rightarrow E$ is non-zero $L$-weakly compact, the operator $S: E \rightarrow E$ is any bounded operator such that $S^{k} T$ is a $L$-weakly compact operator for each $k=1,2, \ldots$ and the norm dual space $E^{\prime}$ have a sublattice $W$ such that lattice isomorphic to $L_{1}(\mu), \mu$ is a measure without atom (we can show like that $W \cong L_{1}(\mu)$ ). If the operator $T: E \rightarrow E$ is a $L$-weakly compact operator then the adjoint operator $T^{\prime}: E^{\prime} \rightarrow E^{\prime}$ is a $M$-weakly compact operator. Also, the restricted operator $T_{\left.\right|_{W} ^{\prime}}: W \cong$ $L_{1}(\mu) \rightarrow E^{\prime}$ is a $M$-weakly compact operator. Again, the adjoint operator $\left(T_{\left.\right|_{W} ^{\prime}}^{\prime}\right)^{\prime}: E^{\prime \prime} \rightarrow W^{\prime}$ is a $L$-weakly compact operator. Since all $L$-weakly compact sets is contained in $\left(W^{\prime}\right)^{a} \cong\left(L_{\infty}(\mu)\right)^{a}=\{0\}$ we have

$$
\left(T_{\left.\right|_{W} ^{\prime}}^{\prime}\right)^{\prime}\left(B_{E^{\prime \prime}}\right) \subseteq\left(W^{\prime}\right)^{a} \cong\left(L_{\infty}(\mu)\right)^{a}=\{0\}
$$

Hence, $\left(T_{\left.\right|_{W} ^{\prime}}^{\prime}\right)^{\prime}=0$ holds and it follows that the adjoint operator $T^{\prime}$ is zero on $W$. Similarly, since $S^{k} T: E \rightarrow E$ is $L$-weakly compact operator for each $k=$ $1,2, \ldots$ we have $T^{\prime}\left(S^{k}\right)^{\prime}\left(f_{0}\right)=0$ for each $k=1,2, \ldots$ and for non zero element $f_{0}$ in $W$. Let us pick a non-zero element $x_{0}$ in $E$ such that $T\left(x_{0}\right) \neq 0$. We note that $S^{k} T\left(x_{0}\right) \neq 0$ for each $k=1,2, \ldots$ since we assume that the operator $S$ is oneto-one. Let $V$ denote subspace generated by the set $\left\{T x_{0}, S T x_{0}, S^{2} T x_{0}, \ldots\right\}$. Hence, we obtain that

$$
\left\langle S^{k} T\left(x_{0}\right), f_{0}\right\rangle=\left\langle x_{0}, T^{\prime}\left(S^{k}\right)^{\prime}\left(f_{0}\right)\right\rangle=\left\langle x_{0}, 0\right\rangle=0
$$

for each $k=1,2, \ldots$. It follows that $f_{0}(y)=0$ for every $y \in \bar{V}$. Thus, $\bar{V} \neq E$ holds and it can be seen that $\bar{V}$ is a non-trivial $S$-invariant closed subspace.

We can consider the Banach lattice $E=\ell_{\infty} / c_{0}$ as an example for Theorem 5. Although $\ell_{1}$ is isomorphic to a subspace of $E$ as a Banach space, $E$ does not have any sublattice such that lattice isomorphic to $\ell_{1}$. Because $E$ is order isometric to the Banach lattice $C(\beta \mathbb{N} \backslash \mathbb{N}), \beta \mathbb{N}$ is Stone-Cech compactification ([9], Remarks 2). So, $E^{\prime}$ does not have any sublattice such that lattice isomorphic to $c_{0}\left([6]\right.$, Theorem 2.4.14). Thus, we see that the dual space $E^{\prime}$ have a sublattice such that lattice isomorphic to $L_{1}[0,1]$ ([6], Theorem 5.4.14).

Theorem 8. Let $E$ be a Banach lattice such that its dual space $E^{\prime}$ have a sublattice such that lattice isomorphic to $L_{1}(\mu), \mu$ is a measure without atom and let the operator $T: E \rightarrow E$ be a positive $L$-weakly compact operator. Then, every positive operator $S: E \rightarrow E$ such that $S^{k} T$ is $L$-weakly compact operator for each $k=1,2, \ldots$ have a non-trivial closed invariant subideal. 
Proof. By using technique in the proof of theorem 2 and theorem 5, it is enough to consider that the closed ideal generated by the set $\left\{T x_{0}, S T x_{0}, S^{2} T x_{0}\right.$, ...\} for a non-zero positive element $x_{0}$ in $E$ such that $T\left(x_{0}\right) \neq 0$.

\section{References}

[1] Y.A. Abramovich, C.D. Aliprantis, An Invitation to Operator Theory, Graduate Texts in Math., AMS, Providence, R.I. (2002).

[2] C.D. Aliprantis, O. Burkinshaw, Positive Operators, Academic Press, New York-London (1985).

[3] N. Aronszajn, K.T. Smith, Invariant subspaces of completely continuous operators, Ann. Of Math., 60(1954), 345-350.

[4] V.I. Lomonosov, Invariant subspaces of the family of operators that commute with a completely continuous operator, Funktsional. Anal. $i$ Prilozhen, 7(1973), No:3, 55-56.

[5] P. Meyer-Nieberg, Über klassen schwach kompakter operatoren in Banachverbänden, Math. Z., 138(1974), 145-159.

[6] P. Meyer-Nieberg, Banach Lattices, Springer-Verlag, Berlin-Heidelberg (1991).

[7] H. Radjavi, P. Rosenthal, Invariant Subspaces, Edition, Dover, Mineola, New York (2003).

[8] H.H. Schaefer, Banach Lattices and Positive Operators, Springer-Verlag, Berlin, New York (1974).

[9] M. Wojtowicz, The lattice copies of $\ell_{1}$ in Banach lattice, Comment. Math. Univ. Carolinae, 42(4)(2001), 649-653.

[10] A.C. Zaanen, Riesz Spaces II, North-Holland Publ. Comp., Amsterdam (1983). 
